\title{
25 Research Soure \\ Feasibility investigation of uniportal video-assisted thoracoscopic anatomical lung resection for pulmonary sequestration
}

\author{
Yungang Sun \\ Nanjing Chest Hospital \\ Feng Shao ( $\square$ doctorshao1982@sina.com ) \\ Qiang Zhang \\ Nanjing Chest Hospital \\ Zhao Wang \\ Nanjing Chest Hospital
}

Research article

Keywords: Uniportal video-assisted thoracic surgery, Pulmonary sequestration, mini-invasive technique

Posted Date: September 8th, 2019

DOI: https://doi.org/10.21203/rs.2.14169/v1

License: (c) (i) This work is licensed under a Creative Commons Attribution 4.0 International License.

Read Full License

Version of Record: A version of this preprint was published at Journal of Cardiothoracic Surgery on May 13th, 2020. See the published version at https://doi.org/10.1186/s13019-020-01126-x. 


\section{Abstract}

Background Uniportal video-assisted thoracic surgery (UVATS) technique has been increasingly used for many thoracic diseases. Whether UVATS has equivalent or better perioperative outcomes for pulmonary sequestration (PS) patients remains controversial. Our study aimed to evaluate the feasibility of UVATS in anatomical lung resection for pulmonary sequestration.

Methods A total of 24 patients with PS including fifteen males and nine females with the mean age of $40.54 \pm 14.49$ (rang, 18-65) years old, who had received completely UVATS anatomical lung resection for PS in Nanjing Chest Hospital between January 2016 and December 2018 were retrospectively reviewed. Referring clinical data were retrieved from hospital records and analyzed.

Results All 24 patients had been treated with the UAVTS approach successfully with no aberrant artery ruptured and no massive hemorrhage occurred, and no patients died during the perioperative period. Overall median surgery time was $102.21 \pm 28.00$ mins (range, $55-150$ mins), the mean blood loss was $94.17 \pm 78.90 \mathrm{ml}$ (range, $10-300 \mathrm{ml}$ ), the mean days of chest tube maintained were $4.21 \pm 2.21$ days (range,1-10days), and the mean postoperative hospitalization days was $5.79 \pm 2.35$ days (range,211 days). All patients were cured, without cough, fever, hemoptysis, and so on, occurring during the average follow-up of $17.42 \pm 2.00$ months (range, 3-35months).

Conclusions Our preliminary results revealed that anatomical lung resection by UVATS is a safe and feasible mini-invasive technique for PS patients, which might be associated with less postoperative pain, reduced paresthesia, better cosmetic results, and faster recovery.

\section{Background}

Pulmonary sequestration (PS) is a rare congenital malformation of the lung, in which an island of the lung parenchyma lacks a normal communication with the tracheobronchial tree and receives arterial blood supply from an aberrant branch of systemic circulation rather than the pulmonary circulation, whereas the venous connection is almost normal via pulmonary veins or systemic circulation [1]. As one of the most common types of congenital abnormality of the lower respiratory tract, PS constitutes $0.15-6.4 \%$ of all congenital pulmonary malformations [2]. The theory of the embryologic basis of PS is still unclear, and one of the most widely accepted explanation is that PS results from the formation of an accessory lung bud caudal to the normal lung bud $[3,4]$. Generally the anomalous arterial blood supply usually comes from single or less multiple branches of the thoracic aorta, followed by the abdominal aorta and intercostal aorta [5]. According to the presence or absence of independent visceral pleura 
covering at the normal lung parenchyma, PS can be divided into two types: intralobar and extralobar pulmonary sequestration [6]. Intralobar pulmonary sequestration (ILS) is often found in the lower lobes, especially in the left lower lobe. However, extralobar pulmonary sequestration (ELS) can appear in many spaces, common in the lower lungs and the spine, occasionally in mediastinum, diaphragm, abdomen. PS can often cause respiratory infection, which will lead to a mass of complications, from common pneumonia, emphysema, and lung abscesses to severe hemoptysis, congestive heart failure, and even malignant transformation $[4,6,7]$. Therefore, this means that it is very important to diagnose the PS correctly. Digital subtraction angiography (DSA) once considered being the gold standard for the diagnosis of PS, because it clearly shows the aberrant vessels. So far, with the advent of non-invasive techniques, such as computed tomography angiography (CTA) has become the most commonly used clinical practice in the diagnosis of PS, DSA has fallen out of favor, because of its ingenuity.

Currently, surgical resection is the best way to treat PS regardless of symptoms, in order to resolve lesions, make a definite diagnosis and prevent future complications [8]. Previously, many surgeons have demonstrated the feasibility of thoracotomy in treating ILS and ELS [6,9]. With the development of VATS surgical technique and sophisticated instruments, VATS has increasingly become the preferred operative procedure in the treatment of PS, which is associated with the potential advantages including less postoperative pain, better cosmetic results, and faster recovery [10-14]. The pursuit for mini-invasive keeps promoting the evolution of VATS from traditional multiple-port to single-port for most VATS resections, and with the progress of VATS experience, UVATS has become technically feasible to perform lung resections. When compared with traditional multiple-port VATS, UVATS was found to result in equivalent perioperative outcomes and reduced postoperative pain and discomfort [15]. Despite these advancements and the popularization of the UVATS technique, lung resection for PS remains a prohibited area. The technical restrictions come from both dense pleural adhesions and the unknown abnormal arteries. Therefore, UVATS in PS had seldom been reported [16-19]. Nevertheless, as far as we know, this is the largest number of patients that report the use of UVATS in the treatment for PS. 
Herein, the present study aimed to share some points for dealing with the abnormal arteries and discussing the safety and feasibility of lung resection by UVATS for patients with PS.

\section{Methods}

\section{Patients selection}

A total of 24 patients with PS including fifteen males and nine females with the mean age of 40.54 \pm 14.49 (rang, 18-65) years old, who had received completely UVATS anatomical lung resection of PS in Nanjing Chest Hospital between January 2016 and December 2018 were retrospectively reviewed (Table 1). All the lesions located in the lower lobe with 1-3 aberrant arteries. Four (16.7\%) in the right lower lobe, 20 (83.3\%) in the left lower lobe. This retrospective study was approved by Nanjing Chest Hospital Review Board and the Ethics Committee. 
Table 1 Clinical data

\begin{tabular}{|c|c|}
\hline Characteristics & Value \\
\hline \multicolumn{2}{|l|}{ Gender } \\
\hline Male & $15(62.5 \%)$ \\
\hline Female & $9(37.5 \%)$ \\
\hline \multicolumn{2}{|l|}{ Age (y) } \\
\hline Mean & $40.54 \pm 4.49$ \\
\hline Range & $18-65$ \\
\hline \multicolumn{2}{|l|}{ Preoperative symptoms } \\
\hline Yes & $21(87.5 \%)$ \\
\hline No & $3(12.5 \%)$ \\
\hline \multicolumn{2}{|l|}{ Types } \\
\hline ILS & $21(87.5 \%)$ \\
\hline ELS & $3(12.5 \%)$ \\
\hline \multicolumn{2}{|l|}{ Locations } \\
\hline Left lower lobe & $20(83.3 \%)$ \\
\hline Right lower lobe & $4(16.7 \%)$ \\
\hline \multicolumn{2}{|l|}{ Origin of aberrant artery } \\
\hline Thoracic aorta & $20(83.3 \%)$ \\
\hline Abdominal aorta & $3(12.5 \%)$ \\
\hline Other & $1(4.2 \%)$ \\
\hline \multicolumn{2}{|l|}{ Number of aberrant artery } \\
\hline 1 & $20(83.3 \%)$ \\
\hline 2 & $3(12.5 \%)$ \\
\hline 3 & $1(4.2 \%)$ \\
\hline \multicolumn{2}{|c|}{ Maximum caliber of aberrant artery(mm) } \\
\hline Mean & $7.50 \pm 3.65$ \\
\hline Range & 4-20 \\
\hline
\end{tabular}

ILS: intralobar pulmonary sequestration; ELS: extralobar pulmonary sequestration.

Patients were selected inclusion in the study based on the following criteria: (i) good cardiopulmonary function without surgical contraindications; (ii) no history of surgery on the chest; (iii) all patients were diagnosed or suspected as PS before surgery, and pathology confirmed as PS; (iv) preoperative symptoms were controlled smoothly; (v) all operations were performed under UVATS and completed by isolating the aberrant artery prioritization; (vi) all patients were written informed consent before operation and approval from the institutional review board at our hospital. Exclusion criteria for this study as follows: (i) unacceptable intraoperative single lung ventilation; (ii) previous history of combined thoracotomy; (iii) diffuse dense adhesions cannot use UVATS; (iv) patients who applied for withdrawal midway.

\section{Preoperative work-up}


The preoperative workup for PS mainly includes two aspects, one was to preoperative diagnosis and then guide surgery, and the other was to control the symptoms of infection. In our institution, the computed tomography angiography (CTA) was suspected for PS, as CTA images could identify the aberrant artery from systemic circulation and guide surgical planning. If necessary, digital subtraction angiography (DSA) or magnetic resonance imaging (MRI) should be performed.

Patients with respiratory symptoms were candidates for initial antibiotic treatment, in order to control the preoperative inflammation and increase the safety of surgery. Of the 24 patients in this study, the main symptoms were expectoration (11 cases, $45.8 \%$ ), followed by cough ( 5 cases, $20.8 \%$ ), fever ( 2 cases, $8.3 \%$ ), chest pain ( 2 cases, $8.3 \%$ ), and hemoptysis (1 case, $4.3 \%)$. Three (12.5\%) patients were asymptomatic and did not need preoperative antibiotics (Table 2).

\begin{tabular}{lc} 
Table 2 Preoperative symptoms \\
\hline Main symptoms & $\mathrm{N}(\%)$ \\
\hline expectoration & $11(45.8 \%)$ \\
cough & $5(20.8 \%)$ \\
fever & $2(8.3 \%)$ \\
\hline chest pain & $2(8.3 \%)$ \\
\hline hemoptysis & $1(4.2 \%)$ \\
\hline asymptom & $3(12.5 \%)$ \\
\hline
\end{tabular}

Lobectomy of the lung lesion located was performed for patients with ILS, however, mass excision of the sequestered lung tissue was performed for all ELS patients. Caliber and number of aberrant arteries were not considered as contraindications to UVATS.

\section{Surgical technique}

All Patients were placed in a lateral decubitus with the arm in the swimming position, which was raised for wide intercostal space, and the operations were performed after a double-lumen endotracheal being inserted. For UVATS, only an approximately $3 \mathrm{~cm}$ single incision was made at the fourth or fifth intercostal space of the anterior axillary line with the inserted plastic 
wound protector (Johnson, New Brunswick, NJ, USA). The vision was obtained through a thirtydegree, $10 \mathrm{~mm}$ video-thoracoscope (Karl Storz, Tuttlingen, Germany).

Before any lung resection was performed, we needed to exclude any significant adhesions in the whole chest cavity carefully. According to the preoperative imaging, proceeded to separate the inferior pulmonary ligament, looking carefully for any aberrant artery, where it was always located in. Hereafter, remove the aberrant arteries depends on some methods. Drawing on our experience, the smaller aberrant artery was first ligated proximally using a not-resorbable suture, then proximally and distally clipped with Hemo-o-Lock clips, and then finally was cut with scissors. However, the bigger aberrant artery was also first ligated proximally using a not-resorbable suture and then divided using a vascular endostapler (Ethicon Endo-Surgery, Inc). Due to the high pressure of aberrant arteries, suture ligation was very necessary to reduce the risk of bleeding. After ensuring that all aberrant arteries were divided, which gave more security to avoid bleeding complications, then the lung resection should be performed. For patients with ILS, a routine dissection that dividing the lobar vein, bronchus, and artery in an order, was a completed lobectomy using the standard uniportal technique [20]. For patients with ELS, determining and dissecting the vein drainage, which was passed through the accessory fissure between the lower lobe and the lesion, and then separated the fissure between the lesion and lower lobe with endostapler. At the end of the operation, the arterial stump was covered with fibrin glue and a single 20th chest tube was used to drain the pleural space via the uniport.

\section{Postoperative management and follow-up}

After surgery, the patient was transferred to the normal surgical ward and with ECG monitoring for one night. And then early removal of the urethra and the ECG monitor, allowing patients to mobilize early, promoting lung recruitment and pleural effusion, which was involved as part of rapid recovery. When there was no air-leakage and fluid drainage was less than $200 \mathrm{ml}$ within 24 hours, the chest drain was removed. Patients were usually discharged the day after removal of the chest drain and were followed up in the outpatient clinic. The 
presence of respiratory infection symptoms, such as cough, fever, hemoptysis, and so on, were assessed.

\section{Statistical analyses}

All data were analyzed using SPSS version 21.0 (SPSS Inc, Chicago, IL, USA). The continuous data were presented as mean \pm standard deviation. The categorical variables were presented as

numbers.

\section{Results}

All the surgeries were carried out using the UAVTS approach successfully with no intraoperative conversions or complications. Also, no aberrant artery ruptured or massive hemorrhage occurred.

All the lesions located in the lower lobe with 1-3 aberrant arteries. Four (16.7\%) in the right lower lobe, $20(83.3 \%)$ in the left lower lobe. 21 (87.5\%) ILS were received lobectomy and 3(12.5\%) ELS were received mass excision. Overall median surgery time was $102.21 \pm 28.00$ mins (range, 55-150mins), the mean blood loss was $94.17 \pm 78.90 \mathrm{ml}$ (range, $10-300 \mathrm{ml}$ ), the mean days of chest tube maintained were 4.21 2.21 days (range,1-10days), and the mean postoperative hospitalization days was $5.79 \pm 2.35$ days (range,2-11days) (Table 3 ). No patients died during the perioperative period and only one patient developed early postoperative complications: asymptomatic pneumothorax. (Table 4)

All patients were diagnosed as PS according to postoperative pathology and were cured, without cough, fever, hemoptysis, and so on, occurring during the average follow-up of 17.42 \pm 2.00 months (range, 3-35months). (Table 3)

\section{Table 3 Operation outcomes}




\begin{tabular}{cc}
\hline Variable & Value \\
\hline Pleural adhesion & $15(62.5 \%)$ \\
No & $9(37.5 \%)$ \\
Surgical resection & $21(87.5 \%)$ \\
Lobectomy & $3(12.5 \%)$ \\
Mass excision & \\
Surgery time(min) & $102.21 \pm 28.00$ \\
Mean & $55-150$ \\
Range & $94.17 \pm 78.90$ \\
Mean & $10-300 \mathrm{ml}$ \\
Range & \\
Meod loss(ml) & $10-450$ \\
Range & $4.21 \pm 2.21$ \\
First day drainage volume(ml) & $1-10 \mathrm{~d}$ \\
Mean & \\
Range & $5.79 \pm 2.35$ \\
Drainage tube maintain(d) & $2-11 \mathrm{~d}$ \\
Mean & $17.42 \pm 2.0$ \\
Range & $3-35$ \\
postoperative hospital stay(d) & \\
Mean & \\
Range &
\end{tabular}

Table 4 Complications

\begin{tabular}{cc}
\hline Variable & Value \\
\hline Intraoperative comlications & \\
Pulmonary congestion & $1(4.2 \%)$ \\
\hline Aberrant artery rupture & 0 \\
Massive hemorrhage & 0 \\
\hline Postoperative complications & \\
\hline Pneumothorax & $1(4.2 \%)$ \\
Pulmonary infection & 0 \\
Bronchopleural fistula & 0 \\
Pleural effusion & 0 \\
Arrhythmia & 0 \\
\hline
\end{tabular}


PS is an uncommon lung tissue that lacks communication with the normal bronchial tree and receives its aberrant artery blood supply from the systemic circulation, most frequently the descending aorta [1]. PS is classified into two main types: ILS and ELS. ILS is the most common variant, accounting for $75-93 \%$ of all PS cases $[6,21,22]$ and $87.5 \%$ in our study. Both two types are more common in lower lobes in the literature [6], and all patients in our study located in lower lobes, especially in the left lower lobe. PS is a congenital and benign disease, but the potential complications are serious, which may include common pneumonia, emphysema, lung abscess, severe hemoptysis, congestive heart failure, and even malignant transformation [23-25]. Therefore, surgical resection of PS is the preferred and definitive treatment of choice after the diagnosis is made.

The conventional surgical procedure is open posterolateral thoracotomy, which is safe and feasible, however, this technique might lead to greater trauma and pain, and patients recover more slowly $[3,26]$. More recently many surgeons have confirmed the feasibility of VATS for PS, because of its minimally invasive, pain relief and quick recovery [10-14]. However, surgeons' pursuit of ultimate minimally invasive goals never stops. At present, UVATS has become an increasingly popular approach for surgical treatment of thoracic diseases, including PS. The potential advantages of UVATS include more intuitive vision, less postoperative pain, reduced paresthesia, and better cosmetic results compared with the conventional multiple-port VATS $[27,28]$. Nevertheless, UVATS remains many difficulties, as they still some controversies regarding the safety and feasibility associated with this technique, including small operating space, instruments interfering with each other and fewer entry points for introducing staplers [15]. In addition to the PS patients, repeated infections lead to dense adhesion of the pleural cavity, and more importantly, the aberrant arteries are very concealed and difficult to handle, all of that cause great challenges for the treatment of PS by UVATS.

In our institute, we have quite a lot of UVATS experience, in addition to conventional lobectomy and segmentectomy, and many complex operations, such as bronchus sleeve lobectomy, double sleeve lobectomy, and trachea resection can be performed, which have overcome some limitations of the UVATS itself. However, to our knowledge, only several previous case reports of UVATS for PS were published [16-19]. The first challenging step is to 
separate the presence of extensive adhesions. The following is to make the identification and closure of the aberrant artery, which is a supply from the systemic circulation. In case of failure to safely remove aberrant arteries, it can lead to fatal catastrophic hemorrhage. In this case, it is necessary and relatively safe to follow the principle of "first abnormal blood vessels, posterior pulmonary blood vessels", priority to closure aberrant arteries.

In this study, anatomical lung resections of PS were carried out using the UAVTS approach successfully with no significant postoperative complications and no perioperative death in all 24 cases. In terms of skills and details during operation, the following conclusions are made: (i) Preoperative preparations must be sufficient, as for patients with symptoms of infection, antibiotics must be used to control the symptoms of infection, because repeated infections not only suggest that patients may have extensive pleural adhesions, but patients in the infected period are prone to postoperative sepsis, it also increases the difficulty of identifying aberrant arteries. In addition, preoperative CTA, if necessary combined with MRI or DSA, to identify the caliber and number of aberrant arteries, is essential for surgical planning. As the principle of "first abnormal blood vessels, posterior pulmonary blood vessels" is followed, it is an indispensable rule to get the basic information of the aberrant artery before surgery. (ii) The importance of a single incision is not negligible, an approximately $3 \mathrm{~cm}$ single incision is made in the fifth intercostal space along the anterior axillary line and slightly backward. Because the PS is basically in the lower lung, and the aberrant arteries are usually located in the inferior pulmonary ligament, and the incision is slightly backward to gain better exposure of the aberrant arteries, which can make the vision more intuitive and increase the safety of the operation. (iii) For most patients with PS, pleural adhesions are present, and 16 of our 24 patients had various kinds of adhesions. For pleural adhesions, we should carefully and alternately use electrocoagulation and ultrasonic scalpel separation, as little as possible lung injury and extensive oozing, less lung air leakage, and postoperative drainage, fully loosening adhesions, mainly increase lung freeness, easy to flip, adjust the angle, get a better surgical vision. Of course, thoracotomy or multiple-port VATS is a wise choice when the adhesions are too dense for dissection by UVATS. (iv) Before any lung resection is performed, the most important step is to find out the aberrant arteries. Combined with the preoperative images and 
clinical experience, the aberrant artery is mainly located in the inferior pulmonary ligament and enters the lung tissue. Note that some uncommon local sources, such as abdominal aorta and intercostal aorta, and so on, in this study, most of the aberrant arteries could be identified by preoperative CTA and other tests, without damage to aberrant arteries, the mean intraoperative blood loss was $94.17 \pm 78.90 \mathrm{ml}$ (range, 10-300ml) and the most bleeding patient was $300 \mathrm{ml}$, mainly due to dense adhesion of the pleural cavity. (v) Drawing on our experience, removal of the aberrant arteries should depend on their caliber and avoid blind clamping, cutting and sewing. The smaller aberrant artery was first ligated proximally using a not-resorbable suture, then proximally and distally clipped with hemo-o-Lock clips, and then finally was cut with scissors. However, the bigger aberrant artery was also first ligated proximally using a not-resorbable suture and then divided using a vascular endostapler. Due to the local infection, lacking of muscle layers and high pressure of aberrant artery, it is easy to rupture and hemorrhage, so suture ligation is very necessary to reduce the risk of bleeding. Following make sure that all aberrant arteries are processed. Sometimes more than one aberrant artery may exist, if there are any omissions, if the pulmonary veins are treated, it will cause the congestion of the lungs to occupy most of the chest, which will interfere with the surgical field and increase the risk. In addition, if the aberrant artery branch left unprocessed, it may encounter unpredictable damage that causes the aberrant arteries to retract to the mediastinum or inside the diaphragm causing uncontrollable bleeding. We strongly recommend that for a wider inferior pulmonary ligament, careful dissection must be done to ensure that the isolated aberrant arteries are not missing. Besides the number of aberrant arteries determined should be consistent with the preoperative images, and any suspect aberrant artery should be treated with caution. In our studies, one patient was found to have rapid hyperemia and swelling of the diseased lung after the inferior pulmonary vein was cutting off. Almost half of the chest cavity was occupied, and the anesthesiologist found that there was also oozing in the double-lumen endotracheal. After careful investigation, small diameter of about $8 \mathrm{~mm}$ was presented in the lower part of the inferior pulmonary vein originated from the descending aorta. After the dissection of this aberrant artery, hyperemia and swelling of the diseased lung and oozing in the double-lumen endotracheal were stopped. (vi) For ILS patients, although literature reported that sublobar resection was feasible, we 
recommend lobectomy to reduce the likelihood of recurrence. We have previously reported that the UVATS lobectomy procedure [20]. For ELS patients; only need a mass excision, but because the anatomical location is close to the esophagus, surgery should be careful to avoid injury to the esophagus, if necessary, stomach tube can be applied to assist operation preoperative or intraoperative. (vii) Last but not least, in the surgical operation surface, especially on the stump of the aberrant arteries, the fibrin glue is covered to reduce the air leakage, exudation and drainage, so that the chest tube can be removed early and the hospital stay can be shortened.

Additionally, the potential limitations of the present study may include a small size of retrospective cases study, and a lack of statistical data analysis with other surgical approaches. In our further study, we would increase the sample size and further investigate the clinical outcomes to confirm the safety and feasibility of UVATS technique for anatomical lung resection of pulmonary sequestration.

\section{Conclusions}

In virtue of the surgical technical advantages, anatomical lung resection for PS patients using the UVATS approach ensured the safety and feasibility of the procedure and might benefit more patients with PS from minimally invasive treatment. Moreover, UVATS approach might be associated with less postoperative pain, reduced paresthesia, and better cosmetic results compared with the conventional thoracotomy or multiple-port VATS approach.

\section{Abbreviations}

UVATS: Uniportal video-assisted thoracic surgery;

PS: pulmonary sequestration;

ILS: intralobar pulmonary sequestration; 
ELS: extralobar pulmonary sequestration;

DSA: Digital subtraction angiography;

CTA: computed tomography angiography;

MRI: magnetic resonance imaging

\section{Declarations}

\section{Acknowledgments}

We thank Dr Chang Gu for his constructive suggestions and comments.

\section{Funding}

Not applicable

\section{Authors' Contributions}

Yungang Sun drafted the manuscript. Qiang Zhang and Zhao Wang participated in the design of the study and performed the statistical analysis. Feng Shao and Yungang Sun conceived of the study, and participated in its design and coordination and helped to draft the manuscript. All authors read and approved the final manuscript.

\section{Ethics approval and consent to participate}

This study was approved by the Ethics Committee of Nanjing Chest Hospital, Nanjing Brain Hospital Affiliated to Nanjing Medical University, and written informed consent was obtained before surgery from all patients. 


\section{Consent for publication}

Not applicable.

\section{Competing interests}

The authors declare that they have no competing interests.

\section{References}

1. Kravitz RM. Congenital malformations of the lung. Pediatr Clin North Am 1994;41:453-72.

2. Li WW, Lee TW, Lam SS, Ng CS, Sihoe AD, Wan IY, Yim AP. Quality of life following lung cancer resection: video-assisted thoracic surgery vs thoracotomy. Chest 2002;122:584-589.

3. Savic B, Birtel FJ, Tholen W, Funke HD, Knoche R. Lung sequestration: report of seven case and review of 540 published cases. Thorax 1979;34:96-101.

4. Corbett HJ, Humphrey GM. Pulmonary sequestration. Paediatr Respir Rev 2004;5:59-68.

5. Lee DI, Shim JK, Kim JH, Lee HY, Yun YK, Chun KJ. Pulmonary sequestration withright coronary artery supply. Yonsei Med J 2008;49:507-8.

6. Wei Y, Li F. Pulmonary sequestration: a retrospective analysis of 2625 cases in China. Eur J Cardiothorac Surg. 2011;40:39-42.

7. Tanaka T, Ueda K, Sakano H, Hayashi M, Li TS, Zempo N. Video-assisted thoracoscopic surgery for intralobar pulmonary sequestration. Surgery. 2003;133:216-8.

8. Singh R, Davenport M. The argument for operative approach to asymptomatic lung lesions. Semin Pediatr Surg 2015;24:187-95.

9. Gezer S, Taştepe I, Sirmali M, Findik G, Türüt H, Kaya S, Karaoğlanoğlu N, Cetin G. Pulmonary sequestration: a single-institutional series composed of 27 cases. J Thorac Cardiovasc Surg 2007;133:955-9. 
10. Shen JF, Zhang XX, Li SB, Guo ZH, Xu ZQ, Shi XS, He JX. Complete video-assisted thoracoscopic surgery for pulmonary sequestration. J Thorac Dis 2013;5:31-5.

11. Wang LM, Cao JL, Hu J. Video-assisted thoracic surgery for pulmonary sequestration: a safe alternative procedure. J Thorac Dis 2016;8(1):31-36.

12. Kestenholz PB, Schneiter D, Hillinger S, Lardinois D, Weder W. Thoracoscopic treatment of pulmonary sequestration. Eur J Cardiothorac Surg 2006;29:815-8.

13. Inoue T, Oizumi H, Nakamura M, Sadahiro M. Port-access thoracoscopic anatomical segmentectomy for pediatric intralobar pulmonary sequestration. Thorac Cardiovasc Surg Rep. $2014 ; 3: 42-4$.

14. Gonzalez D, Garcia J, Fieira E, Paradela M. Video-assisted thoracoscopic lobectomy in the treatment of intralobar pulmonary sequestration. Interact Cardiovasc Thorac Surg. $2011 ; 12: 77-9$.

15. Chang JM, Kam KH, Yen YT, Huang WL, Chen W, Tseng YL, Wu MH, Lai WW, GonzalezRivas D. From biportal to uniportal video-assisted thoracoscopic anatomical lung resection: A single-institute experience. Medicine. 2016;95(40): e5097.

16. Tamura M, Shimizu Y, Hashizume Y. Single-incision video-assisted thoracic resection for extrapulmonary sequestration: a case report. Journal of Cardiothoracic Surgery 2014;9:22.

17. Lin Z-w, Xu S-t, Wang Q. Uniportal video-assisted thoracic lobectomy in a semiprone position for the treatment of a large intralobar pulmonary sequestration. Interact CardioVasc Thorac Surg 2015;21:542-4.

18. Sihoe ADL, Luo Q, Shao G, Li Y, Li J, Pang D. Uniportal thoracoscopic lobectomy for intralobar pulmonary sequestration. Journal of Cardiothoracic Surgery 2016;11:27.

19. Dell'Amore A, Giunta D, Campisi A, Congiu S, Dolci G, Barbera NA, Agosti R, Buia F. Uniportal thoracoscopic resection of intralobar and extralobar pulmonary sequestration. J Vis Surg 2018;4:63.

20. Liu Z, Yang R, Shao F, Pan Y. Modified procedure of uniportal video-assisted thoracoscopic lobectomy with muscle sparing incision. Ann Transl Med 2016;4(19):367.

21. Van Raemdonck D, De Boeck K, Devlieger H, Demedts M, Moerman P, Coosemans W, Deneffe G, Lerut T. Pulmonary sequestration: a comparison between pediatric and adult patients. Eur J Cardiothorac Surg $2001 ; 19: 388-95$. 
22. Liu C, Pu Q, Ma L, Mei J, Xiao Z, Liao H, Liu L. Video-assisted thoracic surgery for pulmonary sequestration compared with posterolateral thoracotomy. J Thorac Cardiovasc Surg 2013;146:557-61.

23. Hazer S, Aydogdu K, Incekara F. Are pulmonary sequestrations innocent enough due to comorbidities? J Clin Anal Med 2016;7:375-9.

24. Fabre OH, Porte HL, Godart FR, Rey C, Wurtz AJ. Long-term cardiovascular consequences of undiagnosed intralobar pulmonary sequestration. Ann Thorac Surg 1998;65:1144-6.

25. Belchis D, Cowan M, Mortman K, Adenocarcinoma arising in an extralobar sequestration: a case report and review of the literature. Lung Cancer 2014;84:92-5.

26. Watine O, Mensier E, Delecluse P, Ribet M. Pulmonary sequestration treated by videoassisted thoracoscopic resection. Eur J Cardiothorac Surg 1994;8:155-6.

27. Gonzalez-Rivas D, Paradela M, Fernandez R, Delgado M, Fieira E, Mendez L, Velasco C, de la Torre M. Uniportal video-assisted thoracoscopic lobectomy: two years of experience. Ann Thorac Surg 2013;95:426-32.

28. Wang BY, Liu CY, Hsu PK, Shih CS, Liu CC. Single-incision versus multiple-incision thoracoscopic lobectomy and segementectomy: a propensity-matched analysis. Ann Surg 2015;261:793-9. 